\title{
The effects of two loading patterns of resistance training (skewed pyramid \& reverse step) on some physical and physiological capabilities of non-athlete
}

\section{men}

\author{
Ali Sayyah®1, Ehsan Asghari®2, Hamid Arazi@3 \\ ${ }^{1}$ Department of Sport Sciences, Faculty of Humanities, University of Zanjan, Zanjan, Iran. ${ }^{2}$ Department of Exercise Physiology, Faculty of \\ Sport Sciences, Ferdowsi University of Mashhad, Mashhad, Iran. ${ }^{3}$ Department of Exercise Physiology, Faculty of Sport Sciences, University of \\ Guilan, Rasht, Iran.
}

\begin{abstract}
The present study aims to investigate the effects of two different loading patterns of resistance training (skewed pyramid \& reverse step) on some physical and physiological capabilities of non-athlete men. For this purpose, 30 non-athlete men with a resistance training experience of less than six months are selected among volunteers and randomly assigned to control $(n=10)$, reverse step loading $(n=10)$, and skewed pyramid loading $(\mathrm{n}=10)$ groups. Their strength, speed, agility, explosive power, muscle volume, and body composition were measured before and after 10 weeks of training. Two-way ANOVA and Tukey post-hoc tests were used for statistical analysis. Results show that in both groups of skewed pyramid and reverse step, the variables of strength, speed, agility, explosive power, and muscle volume have had a significant increase compared to the control group. Also, the body composition of the training groups significantly improved and fat percentage decreased $(p<0.05)$. However, there were more positive changes in thigh volume, lower body strength and body fat percentage in skewed pyramid compared to reverse step $(\mathrm{p}<0.05)$. Generally, it seems that the skewed pyramid pattern has a higher importance and priority than the reverse step pattern in improving both physical and physiological factors, especially muscle strength and muscle volume development of non-athlete men.
\end{abstract}

Keywords: Adaptation, agility, loading pattern, muscle power, strength training.

\section{Introduction}

Resistance training is one of the general exercises recommended for a wide range of people (healthy people, adults and the elderly) as well as patients (cardiovascular and neuromuscular diseases) by the American College of Sports Medicine (ACSM) and the American Heart Association (AHA) (Kraemer \& Ratamess, 2004; Kraemer et al., 2002). The development of maximum power and explosive power, as well as the improvement of the body composition, especially in non-athletes, can have a significant effect on improving one repetition maximum (1RM) as well as performance. Moreover, duration, intensity, volume of training and rest intervals between training shifts and intervals are affected by 1RM of subjects (Hoseini et al., 2016; Kim et al., 2002). Therefore, the development of strength and power is one of the most important components of training programs to prepare for this type of exercise, and therefore, the attention of many researchers and sports' experts has focused on how to improve these factors (Lira et al., 2015; Mirzaei et al., 2010).

When it comes to non-athletes participating in resistance training programs, their training style

A. Sayyah, e-mail: alisayyah@znu.ac.ir

Received: October 13, 2021 - Accepted: December 29, 2021 - Published: December 31, 2021

To Cite: Sayyah, A., Asghari, E., Arazi, H. (2021). The effects of two loading patterns of resistance training (skewed pyramid \& reverse step) on some physical and physiological capabilities of non-athlete men. Turk J Kinesiol, 7(4), 123-131. DOI: 10.31459/turkjkin.1008780 
becomes more important to achieve the expected results. Because the design and planning of the next course of training of these people is mostly based on their 1RM in each movement (Bompa et al., 2002). Studies show that non-athletes, according to their normal physical needs, can use various strength training programs, such as increasing strength, power, hypertrophy, and muscle endurance (Gaeini et al., 2009; Hoseino et al., 2012). Since proper training design is the main factor in successful strength training at any level of readiness (Brandenburg \& Docherty, 2006; Hoseino et al., 2012), designing strength training is of great importance for nonathletes considering various variables, such as load, volume, repetition, rest pattern between courses as well as training loading pattern (Fleck \& Kraemer, 2004). Also, one of the factors that play a role in determining the magnitude of the effect of resistance training is training systems and different loading patterns that play an important role in increasing muscle strength and endurance (Hoseino et al., 2012). In addition, by changing the way of creating physical stress and preventing early adaptations, the patterns provide more thirst for coping with new shocks and a variety of training models, and the body, as an adaptable origin, adapts to environmental stress of exercise with a higher level of physical and physiological function. Various methods have been proposed for loading patterns or adjustment of training courses. The basis of their design and planning is to change the amount of load and resistance, repetitions and finally sets according to the training goals and level of readiness of people. Their morphological diversity can lead to various training achievements in the musculoskeletal area. These include the pyramid, reverse pyramid, double pyramid, flat pyramid, skewed pyramid, and reverse step (Bompa et al., 2002). Findings of some studies show that when the goal of the training program is to increase maximum strength, the use of skewed pyramid method can be effective to create the necessary adaptations. In the reverse step pattern, the load is reduced from step to step, and some Eastern European weightlifters believe that this method addresses physiological needs and can have the desired effect. However, the latter view needs more scientific discussion (Hoseino et al., 2012). Some researchers have also shown in their studies that maximum strength, muscle volume, and power are completed only when the maximum range of motion is used (Kraemer et al., 2002). In some other studies, it is shown that a similar load of 80 to $85 \%$ of $1 \mathrm{RM}$ and beyond has a greater effect on increasing the maximum dynamic power (Bemben et al., 2000). The range of this load causes the use of maximum muscle fiber, which ultimately increases 1RM (Hoseino et al., 2012). Some other researchers observed a similar increase in strength of 1RM and 10RM after comparing the two methods of pyramid training and reverse pyramid (Fish et al., 2003). Some studies have suggested that using a constant training load at each set, despite applying optimal tension to the muscle, may question the high performance of the exercise by limiting training volume, muscle time under tension (TUT), as well as reducing other stimuli, such as fatigue that prevents effective training stimulation (Brown et al., 2015; Shimano et al., 2006). Some other studies in this field, referring to the use of the loading pattern with a gradual increase in load from one set to the next and increase in the number of repetitions in low-load courses, which leads to an increase in active muscle TUT, state that excessive fatigue (fatigue from the accumulation of metabolic by-products $\left(\mathrm{K}^{+}, \mathrm{Cr}, \mathrm{P}_{\mathrm{i}}\right.$, Lactate, $\mathrm{H}^{+}$) may increase exercise effectiveness (Brown et al., 2015). In addition, the use of a variety of loading range each time of resistance training is consistent with the principle of variety in training and maintains the effectiveness of training stimuli (Shimano et al., 2006). Nevertheless, to the best of the researcher's knowledge, few studies have examined the effect of different loading patterns on increasing the physical and physiological components of individuals, especially non-athletes (Brandenburg \& Docherty, 2006). Although it is recommended to perform courses with the maximum load to increase the maximum strength and factors such as power, speed of movement, muscle volume, and etc., according to the principle of variety of training, it seems that how to use loads to create the desired stimulus for trainingshould be of special importance from one set to the next.

Given the importance of these factors in the performance of non-athlete exercises, planning and designing exercises for this group of people, complete disagreement between the results of studies to determine the correct and optimal method of strength training, as well as the scope of use of multiple training protocols to increase muscle volume, strength and endurance, further studies, especially in the field of loading patterns, seem necessary to achieve the defined results. Therefore, the present study aimed to investigate the effect of a 10 -week resistance training 
course with two loading patterns of skewed pyramid and reverse step (as an effective model introduced in the resistance training loading patterns) on some physical and physiological capabilities of non-athletes, such as power, strength, endurance, volume, speed, muscle agility, and body fat percentage.

\section{Methods}

The statistical sample of this study consisted of 30 non-athletes familiar with resistance training with a training history of less than 6 months (mean age of $19.65 \pm 3$ years, height of $176.73 \pm 5.85 \mathrm{~cm}$, and weight of $68.21 \pm 4 \mathrm{~kg}$ ), who had registered in sports clubs equipped with machines and free weights and wanted to start training. Subjects were randomly divided into three groups of 10 (control $(n=10)$ ), reverse step $(n=$ $10)$, and skewed pyramid $(\mathrm{n}=10)$ ). All subjects, while voluntarily participating in the study and all experimental protocols were approved by University of Zanjan Ethics Committee (Approval ID: IR.ZNU.REC.1400.021, Approval Date: 2021-12-13), all methods were carried out in accordance with relevant guidelines and regulations and informed consent was obtained from all participants, had conditions such as no history of disease, no use of exercise supplements or energy boosters at least four months before the protocol, and no exercise outside the program. In this regard, a special health questionnaire (PARQ and you) was used to assess their health. Subjects were also asked to avoid the use of nicotine, alcohol and any stimulants during the days of the study, not to engage in vigorous exercise activities 24 hours before the start of the process, and any changes in diet. The day before the test, the research conditions and how it was performed were explained for subjects, and preliminary measurements (measurement of height and weight, body fat percentage, $1 \mathrm{RM}$, and muscle circumference) were done. Subjects' height was measured with a stadiometer (with a sensitivity of one millimeter), their weight with a scale (Seca model made in South Korea with a sensitivity of $0.1 \mathrm{~kg}$ ), and their body fat mass with an "InBody" model 3.0 made in South Korea (Rozenek et al., 2007). All tests were performed at the same time in the afternoon to prevent the effect of circadian rhythm on the studied variables. Speed was measured through a 40-yard (36 m) test. Subjects' explosive power was assessed using the vertical jump test (Sargent jump test) of Brown \& Weir (2001). After the initial warm-up, each subject performed three test jumps and the fourth jump as the main jump. Muscle volume was calculated using anthropometric method for thigh muscles (quadriceps and hamstrings) according to the method of Housh et al. (1995), and for the arm muscles based on the method described by Frisencho et al. (1974).

Equations used to estimate the cross-sectional area of the thigh muscle set include:

The cross-sectional area of the hamstring muscle: (half of the thigh circumference in $\mathrm{mm} \times 1.08$ ) (skinfold thickness in the anterior thigh area in $\mathrm{mm} \times$ $0.64)-22 / 69$

Thickness of the total cross-sectional area of the thigh muscle: (half of the thigh circumference in $\mathrm{mm} \times 4.68$ ) - (skinfold thickness in the anterior thigh area in $\mathrm{mm} \times$ 2.09) $-80 / 99$

The Cross-sectional area of quadriceps muscle: (half of the thigh circumference in $\mathrm{mm} \times 2.52$ ) - (skinfold thickness in the anterior region of the thigh in $\mathrm{mm} \times$ 1.25) $-45 / 13$

Equations used to estimate arm muscle size:

Arm muscle diameter (mm): [arm circumference $(\mathrm{mm}) \div \pi$ ] - [skinfold thickness of triceps brachii $(\mathrm{mm})]$

Arm muscle circumference (mm): [arm circumference $(\mathrm{mm})]-[\pi \times$ skinfold thickness of triceps brachii $(\mathrm{mm})]$

Arm muscle surface $\left(\mathrm{mm}^{2}\right):[\pi \div 4] \times[($ arm diameter in $\mathrm{mm})^{2}$ ]

The maximum strength of the subjects was measured using the 1RM test by McGuigan et al. (1997). Thus, before the test and after general warmup, 5 repetitions with $30 \%$ (2 minutes rest), 4 repetitions with 50\% ( 2 minutes rest), 3 repetitions with $70 \%$ (3 minutes rest), and 1 repetition with $90 \%$ (3 minutes rest) were performed to warm up. After performing the last set with $90 \%$ of $1 \mathrm{RM}$, the load was added in subsequent set with feedback from the subjects based on the amount of weight lifted in order to obtain 1RM (2.5 to $10 \mathrm{~kg}$ after each successful attempt). To obtain 1RM after determining $90 \%$ of 1RM, three test steps were performed and 4 minutes rest was considered between each attempt (Bemben et al., 2000). Two training programs as skewed pyramid and reverse step were considered for the subjects. In the pyramid training program with skewed loading pattern, which is a modified version of 
the double pyramid pattern, except for the last set when the load is reduced, the load is added regularly and alternately. In this training model, the goal of the last set is to create variety and motivation, because due to the reduction of the load in the last set, the athlete performs repetitions more easily and faster. The repetitions in the skewed pyramid pattern are as follows: 4 repetitions with $75 \% 1 \mathrm{RM}, 4$ repetitions with $80 \% 1 \mathrm{RM}, 2$ repetitions with $85 \% 1 \mathrm{RM}$, and 2 repetitions with $75 \% 1 \mathrm{RM}$ (Figure 1). The second group practiced using the reverse step pattern (4 repetitions with $85 \%$ 1RM, 10 repetitions with $75 \%$ $1 \mathrm{RM}, 4$ repetitions with $85 \% 1 \mathrm{RM}$ and 10 repetitions with $75 \%$ 1RM) (Figure 2). It is worth mentioning that the intensity and repetitions were adjusted so that both training groups had the same training volume and this issue was eliminated as a factor influencing the results. In each training session, the researchers supervised the work of the subjects and once every two weeks, one repetition maximum was tested. According to the amount of weight lifted, a new program was given to the subject to perform the principle of overload.

\section{Statistical Analysis}

Two-way analysis of variance and Tukey's post-hoc test were used to compare the physiological changes after 10 weeks of resistance training. Paired t-test was used for within-group differences. All operations and statistical analysis were performed using SPSS software version 16 . Also, the minimum significance level in this study was considered $\mathrm{p}<0.05$.

\section{Results}

The results of analysis of variance showed that the strength (Table 2), muscle volume (Figure 3 and 4), speed, agility, and anaerobic (explosive) power of the subjects after 10 weeks of training with two skewed pyramid and reverse step loading patterns increased significantly compared to the control group. With decreasing fat percentage, body composition of training groups also improved significantly (Tables 1; $\mathrm{p}<0.05$ ). The results of Tukey test to evaluate the difference between the training and control groups showed that the strength (Table 2) and muscle volume (Figure 3 and 4) observed in both training groups after 10 weeks of training had a significant increase compared to the control group ( $p<0.05)$. Also, the decrease in body fat percentage in the skewed pyramid group was more than the reverse step group (Table $1 ; \mathrm{p}<0.05$ ). There was no significant difference between the two training groups in terms of explosive power. The only significant difference was between the skewed pyramid training group and the control group ( $\mathrm{p}<0.05)$.

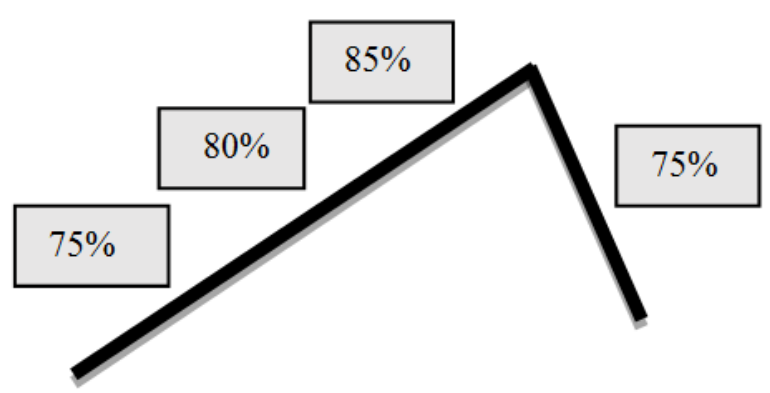

Figure 1. Skewed pyramid loading pattern.

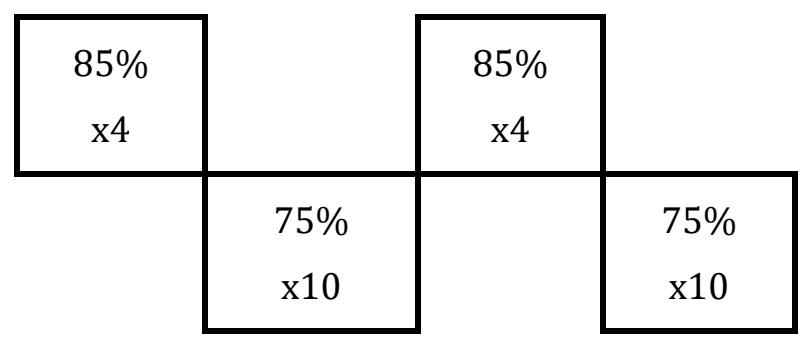

Figure 2. Reverse step loading pattern.

\section{Discussion}

The aim of this study was to investigate the effect of a resistance training course with two different loading patterns (skewed pyramid and reverse step) on the physical and physiological capabilities of non-athletes. The main finding of this study was that after 10 weeks of training in both training groups, significant changes were made in the physical and physiological factors of the subjects compared to the control group. This finding suggests that both training patterns can be used to enhance and improve the physical and physiological factors of nonathletes. However, it seems that the skewed pyramid training pattern has been more effective in improving and developing the strength and muscle volume of non-athlete men in comparison to the reverse step pattern, and from this point of view, it has priority. 


\section{Table 1}

Physical and physiological factors in all of three groups before and after 10 weeks of resistance training with skewed pyramid and reverse step loading patterns.

\begin{tabular}{|c|c|c|c|c|c|c|}
\hline \multirow[t]{2}{*}{ Group Variables } & \multicolumn{2}{|c|}{ Control } & \multicolumn{2}{|c|}{ Skewed pyramid } & \multicolumn{2}{|c|}{ Reverse step } \\
\hline & Pre-test & Post-test & Pre-test & Post-test & Pre-test & Post-test \\
\hline Weight (kg) & $71.34 \pm 3.01$ & $73.91 \pm 2.85$ & $65.2 \pm 2.84$ & $69.7 \pm 3.25 *+$ & $66.11 \pm 3.25$ & $68.5 \pm 4.33 *$ \\
\hline Body fat (\%) & $18.21 \pm 1.34$ & $20.04 \pm 1.5$ & $18.41 \pm 1.77$ & $15.62 \pm 1.02 * \dagger$ & $19.81 \pm 1.85$ & $17.05 \pm 2.44 *+$ \\
\hline Speed (40 yard in s) & $5.65 \pm 1.02$ & $5.57 \pm 1.3$ & $5.45 \pm 1.34$ & $5.04 \pm 1.66 *+$ & $5.61 \pm 1.8$ & $4.85 \pm 1.5^{* \dagger}$ \\
\hline Explosive power $(\mathrm{cm})$ & $43.2 \pm 2.11$ & $41.8 \pm 2.03$ & $46.35 \pm 2.85$ & $53.5 \pm 3.01 *+$ & $44.3 \pm 2.5$ & $52.11 \pm 3.02 *+$ \\
\hline Agility (Illinois) (s) & $15.74 \pm 0.42$ & $15.60 \pm 0.55$ & $14.57 \pm 0.57$ & $14.1 \pm 0.84 *+$ & $14.95 \pm 0.5$ & $14.52 \pm 1.01 *+$ \\
\hline
\end{tabular}

\section{Table 2}

Comparison of strength in upper and lower body exercises among the three groups before and after 10 weeks of resistance training with skewed pyramid and reverse step loading patterns.

\begin{tabular}{|c|c|c|c|c|c|c|}
\hline \multirow{2}{*}{ Group Variables } & \multicolumn{2}{|c|}{ Control } & \multicolumn{2}{|c|}{ Skewed pyramid } & \multicolumn{2}{|c|}{ Reverse step } \\
\hline & Pre-test & Post-test & Pre-test & Post-test & Pre-test & Post-test \\
\hline Bench press (kg) & $45.13 \pm 3.31$ & $45.91 \pm 4.1$ & $67.15 \pm 4.23$ & $76.54 \pm 3.67^{*+}$ & $62.5 \pm 3.84$ & $74.6 \pm 3.43^{*}+\ddagger$ \\
\hline Arm curl (kg) & $25.8 \pm 2.84$ & $26.1 \pm 2.05$ & $35.3 \pm 3.55$ & $39.1 \pm 4.2 *+$ & $33.2 \pm 4.14$ & $40.2 \pm 3.84^{*}+\ddagger$ \\
\hline Lat pull down (kg) & $86.5 \pm 3.41$ & $91.62 \pm 4.22$ & $103.4 \pm 3.1$ & $110.12 \pm 3.3^{*+}$ & $106.6 \pm 4.9$ & $117.3 \pm 5.24^{*}+\ddagger$ \\
\hline Leg press (kg) & $191.53 \pm 4.65$ & $210.58 \pm 3.93$ & $233.5 \pm 4.5$ & $297.8 \pm 4.74 * \dagger \neq$ & $241.36 \pm 5.35$ & $282.9 \pm 5.5^{*+}$ \\
\hline Leg extension $(\mathrm{kg})$ & $33.18 \pm 4.1$ & $34.54 \pm 2.97$ & $45.2 \pm 3.4$ & $57.6 \pm 3.62 * \dagger \ddagger$ & $45.3 \pm 3.26$ & $51.64 \pm 3.7^{*+}$ \\
\hline Leg Curl (kg) & $31.36 \pm 3.7$ & $25.28 \pm 3.11$ & $35.1 \pm 3.7$ & $53.4 \pm 4.18^{*}+\ddagger$ & $37.95 \pm 2.87$ & $48.71 \pm 3.5^{*}+$ \\
\hline
\end{tabular}

+ Significant difference between pre-test and post-test $(p<0.05)$; * Significant difference compared to the control group ( $p<0.05)$; $¥$ Significant difference between skewed pyramid and reverse step $(P<0.05)$.

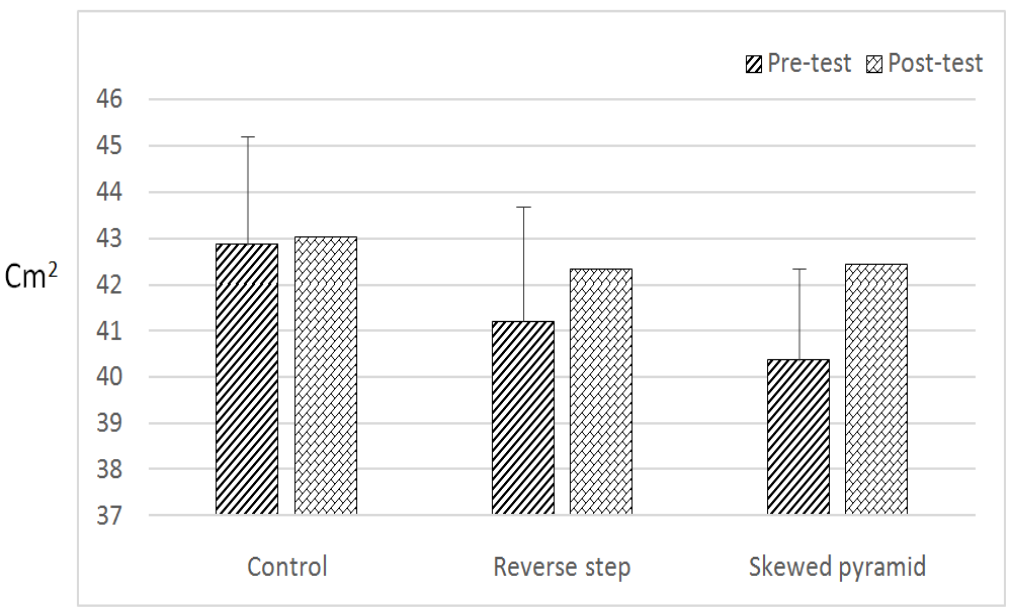

Figure 3. Thigh muscle volume in the three groups in the pre- and post-test stages. 


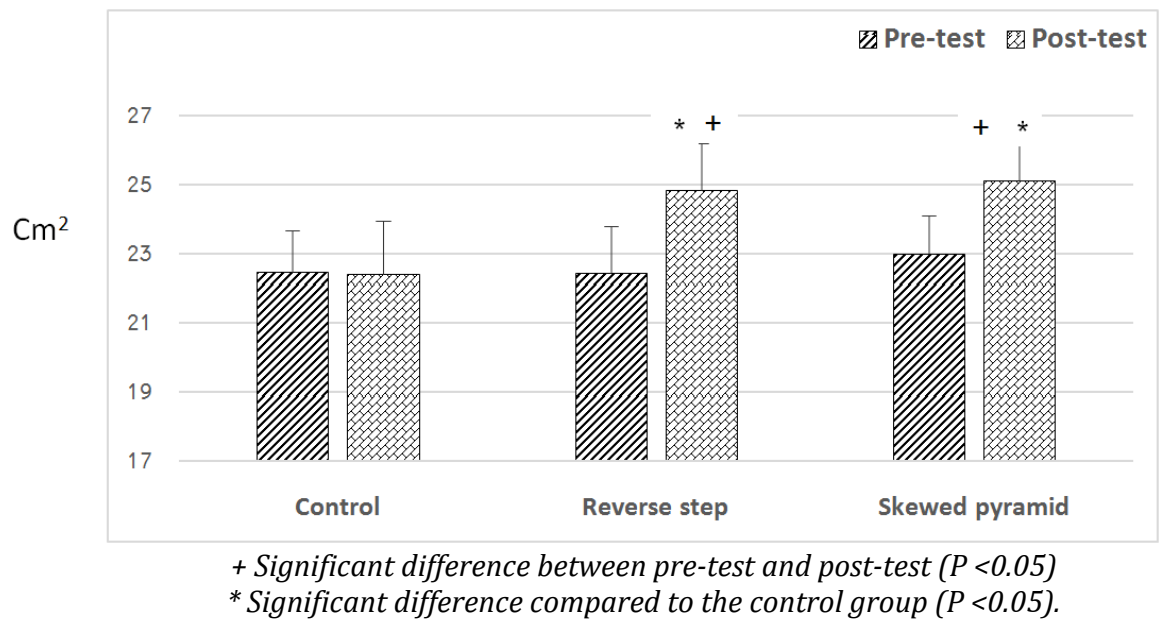

Figure 4. Arm muscle volume in the three groups in the pre- and post-test stages.

Accordingly, the design and selection of resistance exercises for beginners, who are at the beginning of regular and serious sports activities, is of great importance and can be associated with achieving effective physical and physiological achievements in the specialized performance of sports, especially in men. Also, the person may achieve the results defined for him in a shorter time. This can also determine the motivation and will of the person to continue sports activities with specific goals. In other words, the person takes more and faster steps to reach the next stages of training and other expected outcomes. But the question is which of the loading patterns can be more efficient in these factors. Some studies have suggested the possibility of reverse step loading with respect to the maximum load in the first stage compared to pyramid patterns as a model for greater fatigue and reduced strength and muscle volume (Paulsen et al., 2003). However, since part of the findings of this study is that this model also increases the strength and muscle volume of novice subjects compared to the control group, it seems that the use of this method may cause muscle overcompensation or recall of more motor units, ultimately stimulating an increase in strength and muscle volume (Mangine et al., 2015). The findings of this section are in line with the findings of Paulsen et al. (2003). However, when comparing this pattern with the skewed pyramid pattern, it is clear that the skewed pyramid pattern causes a more significant increase in strength and muscle volume than the reverse step pattern. This indicates that the skewed pyramid pattern is more efficient than the reverse step pattern. The main justification for this finding is that in general, non- athletes are not very familiar with strength activities and resistance training loads, and applying the maximum load in the first stages of training in areverse step pattern can reducethe efficiency of this model compared to skewed pyramid pattern. This is because the skewed pyramid pattern follows the principle of training characteristics (gradual increase of load) and the better response of non-athletes to this training pattern may be due to this. Rooney et al. (1994) emphasized the use of a pyramid pattern, which is consistent with the findings of this study, by stating that when the athlete is tired, more units are used and therefore, these stimuli increases strength after muscular activities. It is worth noting that the study of these researchers was conducted on athletes, while the subjects of this study were non-athletes (Rooney et al., 1994).

On the other hand, the use of reverse step pattern that starts with the maximum load and gradually reduces the training load, can be considered as a training model that is associated with activity recovery. This is of great importance for non-athletes, for whom continuing exercise with higher load accelerates fatigue, even if it is from a psychological point of view, and hinders the continuity of activity. The findings of this study indicate that the two training groups had a significant improvement in physiological factors, especially strength, compared to the control group. These changes are evident in some of the findings in the upper extremity when using the reverse step pattern, which can show a better effect of this pattern in comparison to the skewed pyramid pattern in raising this factor in the upper extremities. Of course, since the subjects in this study were non- 
athletes, this may be different for athletes and trainees. In this regard, Brown et al. (2015) suggested that the response to training interference, according to the level of training of the subjects, does not always follow the principle of training characteristics. Moreover, for people with low levels of power, improvement in the force-speed relationship may occur without consideration of the load and the method used, which is consistent with the results of this study (Brown et al., 2015). Fleck \& Kraemer (2004) reported that gaining strength through different training protocols may be different in one muscle group than in another. In support of this theory, Paulsen et al. (2003) observed that the muscles of the lower body showed a greater increase in response to moderate-volume resistance training compared to low-volume resistance training. While the response of upper body muscles to resistance training with moderate and low volume was not different (Paulsen et al., 2003). However, no conclusive reason can be given for the different responses of the upper and lower body muscle groups to two different training protocols. The small number of samples in the training groups may be a major reason for such an outcome. Examining the same results for the lower extremities showed the changes caused by the skewed pyramid pattern, indicating the different effects of different training loading patterns on different muscle groups. The findings of this part of the study are inconsistent with the findings of Kraemer \& Ratamess (2004), who used three different training loads and obtained positive results in increasing the strength of 1RM and did not observe significant differences between different groups. These results were consistent with the findings of Campos et al. (2002), who evaluated the effect of 8 weeks of resistance training on untrained men in three groups of low repetition, moderate repetition and high repetition. In justification of these findings, it can be said that the lower extremities, with more muscle volume and more familiarity with training loads and pressures (bearing body weight and performing more daily activities), better respond to the skewed pyramid pattern that gradually increases the training load. This is because they are ready to increase the training load (Hoseino et al., 2012). On the other hand, the upper extremities, which has less muscle volume and familiarity with training pressure than the lower limb, may respond better to the activity model, which reduces the exercise load from step to step. In this regard, some Eastern European weightlifters believe that this method addresses physiological needs, which is in line with the findings of this study (Bompa et al., 2002).

Changes in muscle volume using different training patterns showed that the skewed pyramid pattern was more efficient than the reverse step pattern. Although muscle volume increased in both the lower and upper limbs during the use of both training patterns, this increase was more significant regarding the skewed pyramid pattern in different sections. Since the number of sets (4 sets), the total number of repetitions (28 repetitions), and the TUT ( 84 seconds) were the same in both groups, one of the influential factors in relation to this phenomenon may be changes in training load, i.e. the average load applied during one set and different times of activity. As mentioned, in the pyramid pattern, the changes in training load are according to the principle of gradual increase of exercise load (75\%, 80\%, 85\% and 75\%) and this method of applying load may accelerate the process of muscle hypertrophy compared to the reverse step pattern. The findings of this part of the study are in line with the findings of Mirzaei et al. (2010).

\section{Conclusions}

Considering the above and the sensitivity of the training program for non-athlete men, it seems that both training models are effective in improving the physical and physiological factors of these population. But the skewed pyramid pattern is especially important in the development of muscle strength as well as the development of muscle volume over the reverse step pattern. However, this is not the case with gaining strength in the upper limbs of the subjects in the present study, and the reverse step pattern may be more efficient. This highlights the importance of specific use of each pattern in the development of specific factors.

\section{Acknowledgements}

The authors express their gratitude to all the participants and all those who sincerely participated in this research. 


\section{Conflict of Interest}

There is no any conflict of interests among the authors.

\section{References}

Bemben, D. A., Fetters, N. L., Bemben, M. G., Nabavi, N., \& Koh, E. T. (2000). Musculoskeletal responses to highand low-intensity resistance training in early postmenopausal women. Med Sci Sports Exerc, 32, 19491957.

Bompa, T. (1999). Periodization training for sports: Program for peak strength in 35 sports. Champaign, IL: Human Kinetics.

Bompa, T., Pasquale, M., \& Cornacchia, L. (2002). Serious strength training. Champaign, IL: Human Kinetics.

Brandenburg, J. \& Docherty, D. (2006). The effect of training volume on the acute response and adaptations to resistance training. Int J Sports Physiol Perform, 1, 108121.

Brown, W. M., Davison, G. W., McClean, C.M., \& Murphy, M. H. (2015). A systematic review of the acute effects of exercise on immune and inflammatory indices in untrained adults. Sports Medicine, 1, 35, 1-10.

Brown, L. E., \& Weir, J. P. (2001). ASEP procedures recommendation, I: accurate assessment of muscular strength and power. JEPonline, 4(3), 1-21.

Campos, G. E., Luecke, T. J., Wendeln, H. K., Toma, K., Hagerman, F. C., Murray, T. F., Ragg, K. E., Ratamess, N. A., Kraemer, W. J., \& Staron, R. S. (2002). Muscular adaptations in response to three different resistancetraining regimens. Specificity of repetition maximum training zones. Eur J Appl Physiol, 88, 50-60.

Fish, D. E., Krabak, B. J., Johnson-Greene, D., \& DeLateur, B. J. (2003). Optimal resistance training: Comparison of DeLorme with Oxford techniques. Am J Phys Med Rehabil, 82(12), 903-909.

Fleck, S. J., \& Kraemer, W. J. (2004). Designing Resistance Training Programs. Champaign, Il: Human Kinetics.

Forghani Ozrudi, M., Shob, S. F., \& Aliabadi, S. R. (2014). Comparison of three methods of weight training to increase the power of the male students of Mazandaran University of Science and Technology. Int J Appl Exerc Physiol, 3 (1), 53-59.

Gaeini, A., Arazi, H., \& Ismail, J. (2009). Comparision of two methods of weight training (pyramid and Oxford) on pectoral muscle strength in novice athletes. Harekat. 35 (1), 129-141.
Hoseini, Y., Mirzaei, B., \& Arazi, H. (2016). Effect of four weeks of detraining after strength training with two different loading patterns on hormonal and physiological factors in young wrestlers. Exercise Physiology, 30 (2), 65-84.

Hoseini, Y., Mirzaei, B., \& Nemati, G. H. (2012). The effect of strength training with two different loading pattern (double pyramid \& reverse step loading) on some physiological capabilities wrestling's young. Exercise physiology, 16(4), 151-166.

Hoseino, F., Mohebbi, H., Rahmaninia, F., \& Damirchi, A. (2012). Comparison of the effect of resistance training (flat and double pyramid pattern) on some physical fitness and anthropometric indices in young elite soccer players. Metabolism and Exercise Journal, 2(1), 73-82.

Kim, P. S., Mayhew, J. L., Peterson, D. F. (2002). A modified YMCA bench press test as a predictor of 1 repetition maximum bench press strength. J Strength Cond Res, 16, 440-445.

Kraemer, W. J., \& Ratamess, N. A. (2004). Fundamentals of resistance training: Progression and exercise prescription. Med Sci Sports Exerc, 36(4), 674-688.

Kraemer, W.J, Ratamess, N. A., \& French, D. N. (2002). Resistance training for health and performance. Current Sports Medicine Reports, 1, 165-171.

Lira, F. S., Panissa, V. L. G., Julio, U. F., \& Franchini, E. (2015). Differences in metabolic and inflammatory responses in lower and upper body high-intensity intermittent exercise. Eur J Appl Physiol, 115, 1467-1474.

Mangine, G. T., Hoffman, J., Gonzalez, A., Townsend, J., \& Wells, A. (2015). The effect of training volume and intensity on improvements in muscular strength and size in resistance-trained men. Physiological Reports, 3, 1-17.

Mirzaei B, Mehrabani J., Azizi M, Barjaste A. (2010). The effect of flat pyramid and skewed pyramid loading pattern on maximal strength, muscle mass and lower muscle power. Research in Sports Sciences, 8, 84-71.

Paulsen, G., Mykelstad, D., \& Raastad, T. (2003). The influence of volume of exercise on early adaptations to strength training. J Strength Cond Res, 17, 115-120.

Rooney, K. J., Herbert, R. D., \& Balnave, R. J. (1994). Fatigue contributes to the strength training stimulus. Med Sci Sports Exerc, 26, 1160-1164.

Rozenek, R., Funato, K., Kubo, J., Hoshikawa, M., \& Matsuo, A. (2007). Physiological response to interval training sessions at velocities associated with $\mathrm{VO}_{2} \max$. J Strength Cond Res, 21, 188-192.

Shimano, H., Shimano, T., Kraemer, W. J., Spiering, B. A., Volek, J. S., Hatfield, D. L., Silvestre, R., Vingren, J. L., Fragala, M. S., Maresh, C. M., Fleck, S. J., Newton, R. U., 
Spreuwenberg, L. P., \& Häkkinen, K. (2006), Relationship between the number of repetitions and selected percentage of $1 \mathrm{Rm}$ in trained and untrained men. $J$ Strength Cond Res, 34, 1271-1275. 